\title{
Fat sub-Riemannian symmetric spaces: the nilpotent case
}

\author{
D.M. Almeida and E. Falbel
}

\begin{abstract}
In this paper we start the classification of strongly bracket-generated subsymmetric spaces. We prove a structure theorem and analyze the nilpotent case.
\end{abstract}

\section{Introduction}

Sub-Riemannian manifolds are manifolds equipped with a non-integrable distribution which, in its turn, is equipped with a metric. Symmetric spaces have an important role in Riemannian geometry and therefore it is interesting to consider a generalization of them in the context of sub-Riemannian geometry as given in [18]. One hopes to obtain classification results by imposing restrictions on the distribution underlining the sub-Riemannian structure. For instance, in the case of sub-Riemannian structures on contact manifolds, sub-symmetric spaces were analyzed in [5], [10].

A natural and simplifying condition on the distribution is that of strongly bracket generation. It means that given a non-vanishing horizontal vector field (that is, at each point the vector field belongs to the distribution), its brackets with other horizontal vector fields generate the full tangent space of the manifold. Strongly bracket generating distributions are also called fat distributions and the first author started a study of the corresponding sub-symmetric spaces in [1]. In particular, from the class of Riemannian symmetric spaces called Wolf spaces it is constructed in [1] a class of sub-symmetric spaces with a distribution which is strongly bracket generated and of codimension three. But the full classification of sub-symmetric spaces with fat distributions of codimension three is not yet achieved.

Key words and phrases: sub-Riemannian geometry, sub-symmetric space, fat distribution, two-step nilpotent Lie algebra.

2010 Mathematics Subject Classification: 53C17. 
The simplest Riemannian symmetric space is Euclidean flat space and it also appears as the tangent space of all symmetric spaces. The analogue of that flat space in the case sub-Riemannian geometry is given by nilpotent groups. For a general theory of tangent spaces in sub-Riemannian geometry see [2]. The understanding of nilpotent symmetric spaces is, therefore, a preliminary step to the classification of general sub-symmetric spaces. Strongly bracket generated nilpotent groups are also called fat or regular. In this paper we classify regular nilpotent sub-symmetric spaces satisfying certain natural conditions.

The results in the paper include a structure theorem (Theorem 3) for sub-symmetric spaces and Theorem 4 which states that nilpotent fat sub-symmetric spaces are always two step nilpotent groups. The classification is therefore reduced to 2-step nilpotent algebras where a description is given in Example 1. But their classification is incomplete, see [16] for an up to date on fat 2-step nilpotent Lie algebras. In particular, a complete classification exists when the center of the nilpotent group is two dimensional (see [11], [16]).

Two step nilpotent groups with a left invariant metric have been studied in the context of Riemannian geometry. In particular, writing a two step nilpotent Lie algebra with an invariant metric $\left\langle\cdot, \cdot>\right.$ as $\mathfrak{n}=\mathfrak{z} \oplus \mathfrak{z}^{\perp}$ with $\mathfrak{z}$ central we introduce, as in [13], the following operator

$$
J: \mathfrak{z} \rightarrow s o\left(\mathfrak{z}^{\perp},<\cdot, \cdot>\left.\right|_{\mathfrak{z}^{\perp}}\right),
$$

defined by

$$
<J_{Z} X, Y>\left.\right|_{\mathfrak{z}^{\perp}}=<Z,[X, Y]>\left.\right|_{\mathfrak{z}}, \quad \forall X, Y \in \mathfrak{z}^{\perp}, \forall Z \in \mathfrak{z} .
$$

Two special cases are worth mentioning. The first one arises when $J_{Z}^{2}=$ $-|Z|^{2} I d(\forall Z \in \mathfrak{z})$ and, in this case, the Lie algebra is called of $H$-type. The second one arises when $J$ is a Lie algebra homomorphism for a certain compact Lie algebra structure on $\mathfrak{z}$ and, in this case, the Lie algebra is called of Rep-type (see [9], [15], [16]). Nilpotent algebras of $H$-type are classified in [3], [4], [13]. The case of fat nilpotent Rep-type are also easily classified. It turns out that they all have either one dimensional or three dimensional center (see [16]). The intersection of these two classes of 2-step nilpotent groups corresponds to Heisenberg groups and its quaternionic analogues.

The Riemannian geometry of these two step nilpotent groups (see [8] for a clear account) can be related to their sub-Riemannian geometry. We compute an adapted connection as defined in [1] and show that these symmetric spaces with the fat condition are flat in the sense of sub-Riemannian geometry. 
As a final remark we recall the result in [7] that every fat 2-step 7-dimensional nilpotent group with center of dimension 3 is, in fact, the Quaternionic Heisenberg group thus providing a classification of fat nilpotent sub-symmetric spaces of dimension seven with codimension three distributions.

\section{Sub-symmetric spaces}

\subsection{Sub-Riemannian manifolds}

In this short section, we define the geometric objects motivating the study of certain Lie algebras with symmetry.

Let $M$ be a smooth manifold, and $T_{x} M$ denote the tangent space at a point $x \in M$. A distribution $\mathcal{D}$ defined on $M$ is called fat or strongly bracket generated if for each $x \in M$ and each local section $Z$ of $\mathcal{D}$ near $x$ with $Z(x) \neq 0$, we have

$$
T_{x} M=\mathcal{D}_{x}+\left\{[Z, W]_{x}: W \text { varies over all sections of } \mathcal{D}\right\}
$$

In terms of the Levi form

$$
\mathcal{L}: \mathcal{D} \times \mathcal{D} \longrightarrow T M / \mathcal{D}, \quad \text { defined by } \quad \mathcal{L}_{x}(v, w)=[V, W]_{x} \bmod \mathcal{D}_{x}
$$

where $V, W$ are extensions of $v, w$ to sections of $\mathcal{D}$, the fat condition means that for each $x \in M$ and each nonzero vector $v \in \mathcal{D}_{x}$ the linear operator $\mathcal{L}_{x}(v, \cdot)$ is surjective. If the distribution has codimension one, the fat condition is equivalent to the contact condition.

A sub-Riemannian manifold is a triple $(M, \mathcal{D}, g)$ where $M$ is a smooth manifold, $\mathcal{D}$ is a smooth distribution defined on $\mathrm{M}$ (i.e. $\mathcal{D}$ is a subbundle of the tangent bundle $T M)$ and $g$ is a Riemannian metric defined on $\mathcal{D}$.

A local isometry between two sub-Riemannian manifolds $(M, \mathcal{D}, g)$ and $\left(M^{\prime}, \mathcal{D}^{\prime}\right.$, $\left.g^{\prime}\right)$ is a diffeomorphism between open sets $\psi: U \subset M \rightarrow U^{\prime} \subset M^{\prime}$ such that $\psi_{*}(\mathcal{D})=\mathcal{D}^{\prime}$ and $\psi^{*} g^{\prime}=g$. If $\psi$ is globally defined, we say that $\psi$ is an isometry.

A sub-Riemannian homogeneous space is a sub-Riemannian manifold $(M, \mathcal{D}, g)$ which admits a transitive Lie group of isometries acting smoothly on $M$.

The general definition of sub-Riemannian symmetric spaces was given in [18].

Definition 1. A sub-Riemannian symmetric space or sub-symmetric space, for short, is a homogeneous sub-Riemannian manifold $(M, \mathcal{D}, g)$ such that, for every point $x_{0} \in M$, the isotropy is compact and, moreover, there is an involution which is an isometry [resp., a local isometry] $\psi$, called the sub-symmetry at $x_{0}$, with $\psi\left(x_{0}\right)=x_{0}$ and $\left.\psi_{*}\right|_{\mathcal{D}_{x_{0}}}=-1$. 
Remark 1 . If $\mathcal{D}$ is a fat distribution we do not need the homogeneity condition in the definition of sub-symmetric spaces. This follows from the existence of the sub-symmetry at all points (see [1]).

\subsection{Sub-symmetric Lie algebras}

In this section we set the description of sub-symmetric spaces in the Lie algebra level. The relation to sub-Riemannian and sub-symmetric spaces will be given in the next section. We recall first the definition of involutive algebras which appear in the study of symmetric spaces (see [14] for an overview):

Definition 2. An involutive Lie algebra (IL-algebra, for short) is a pair $(\mathfrak{g}, \sigma)$ where $\mathfrak{g}$ is a (real) Lie algebra and $\sigma$ is an involutive automorphism of $\mathfrak{g}$. Then there is a canonical decomposition $\mathfrak{g}=\mathfrak{g}^{+} \oplus \mathfrak{g}^{-}$into the \pm 1 -eigenspaces of $\sigma$.

In the case of sub-Riemannian geometry we specify more conditions.

Definition 3. A sub-orthogonal IL-algebra (sub-OIL algebra, for short) is given by the data $(\mathfrak{g}, \sigma, \mathfrak{p}, \mathfrak{k}, B)$ where

1. $\mathfrak{g}$ is a (real) Lie algebra and $\sigma$ is an involutive automorphism of $\mathfrak{g}$. We note the canonical decomposition $\mathfrak{g}=\mathfrak{g}^{+} \oplus \mathfrak{g}^{-}$into the \pm 1-eigenspaces of $\sigma$.

2. $\mathfrak{p} \subset \mathfrak{g}^{-}$.

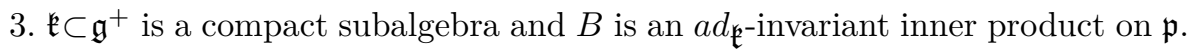

4. $\mathfrak{g}$ is generated as a Lie algebra by $\mathfrak{p}$ and $\mathfrak{k}$.

5. The adjoint action of $\mathfrak{k}$ on $\mathfrak{p}$ is effective.

We define next the Levi form

Definition 4. Consider a sub-OIL-algebra $(\mathfrak{g}, \sigma, \mathfrak{p}, \mathfrak{k}, B)$. The Levi form $\Theta: \mathfrak{p} \times$ $\mathfrak{p} \rightarrow \mathfrak{g}^{+} / \mathfrak{k}$ is defined by

$$
\Theta(X, Y)=[X, Y] / \mathfrak{k}
$$

We will consider in the following algebras such that $\Theta$ is surjective. From now on we prefer to write $\mathfrak{h}=\mathfrak{g}^{+}$. We will also impose that $\mathfrak{p}=\mathfrak{g}^{-}$, although this follows from definitions in some situations. In this case we prefer to drop the $\mathfrak{p}$ in the notation of the sub-OIL-algebra.

Definition 5. Consider a sub-OIL-algebra $(\mathfrak{g}, \sigma, \mathfrak{p}, \mathfrak{k}, B)$. We say the algebra is fat if

$$
\iota_{X} \circ \Theta: \mathfrak{p} \longrightarrow \mathfrak{h} / \mathfrak{k}
$$

is surjective for every non-zero $X \in \mathfrak{p}$. 
Remark 2. We observe that the requirement that $\iota_{X} \circ \Theta$ be surjective in Definition (5) imposes a restrictive condition on the dimension of $\mathfrak{p}$. Let $d=\operatorname{dim} \mathfrak{h} / \mathfrak{k}$, then if $d=1$ this is the contact condition and then dimension of $\mathfrak{p}$ is even [10]; if $d \geq 2$, from ([17], Proposition 5), we have that the dimension of $\mathfrak{p}$ is a multiple of 4 and greater than or equal to $d+1$.

Example 1. Let $(\mathfrak{p}, B)$ be a real vector space with inner product, $\mathfrak{h}$ a real vector space, and let $[\cdot, \cdot]: \mathfrak{p} \times \mathfrak{p} \rightarrow \mathfrak{h}$ be a skew-symmetric surjective bilinear map. Then $(\mathfrak{g}=\mathfrak{p} \oplus \mathfrak{h}, \sigma, \mathfrak{k}=0, B)$ is a two-step nilpotent sub-OIL algebra where the bracket of vectors on $\mathfrak{g}$ is the extension of the bilinear map $[\cdot, \cdot]$ given by $[\mathfrak{p}, \mathfrak{h}]=0,[\mathfrak{h}, \mathfrak{h}]=0$, and the involution $\sigma: \mathfrak{g} \rightarrow \mathfrak{g}$ is defined by $\sigma(X)=-X$ for $X \in \mathfrak{p}, \sigma(X)=X$ for $X \in \mathfrak{h}$. The condition such that $i_{X} \circ[\cdot, \cdot]: \mathfrak{p} \rightarrow \mathfrak{h}$ is onto for all $X \neq 0 \in \mathfrak{p}$ defines then that $(\mathfrak{g}=\mathfrak{p} \oplus \mathfrak{h}, \sigma, \mathfrak{k}=0, B)$ is a two-step nilpotent sub-OIL fat algebra.

\subsection{Correspondence between fat sub-symmetric space and sub-OIL- algebra}

Now, we show the relation between the Lie algebra to the homogeneous space with a sub-Riemannian structure [1].

As in the contact case, to a point $x_{0}$ in a sub-symmetric space $(M, \mathcal{D}, g)$ equipped with a fat distribution we may associate a linear object, its fat suborthogonal Il-algebra. In fact, we take $\mathfrak{g}$ to be the Lie algebra of the group of all sub-Riemannian isometries of $M, \mathfrak{k}$ to be the subalgebra corresponding to the isotropy subgroup at $x_{0}, \sigma=A d_{\psi}$, where $\psi$ is the sub-symmetry at $x_{0}$. Now the differential of the projection $\pi: G \rightarrow M$, given by $\pi(\varphi)=\varphi\left(x_{0}\right)$, identifies $\mathcal{D}_{x_{0}}$ with $\mathfrak{p}$, and we take $B$ to be the inner product $g_{x_{0}}$ lifted to $\mathfrak{p}$ by $\pi_{*}$. Moreover, the fat condition is equivalent to the surjection of the linear map $\Theta_{X}$ for $X \in \mathfrak{p}$. Since $M$ is homogeneous, its sub-OIL algebra is the same at all points.

Conversely, given a fat sub-OIL algebra $\left(\mathfrak{g}^{\prime}, \sigma^{\prime}, \mathfrak{k}^{\prime}, B^{\prime}\right)$ it defines a simply-connected sub-symmetric space with a strongly bracket generating distribution as follows. Let $\widetilde{G}$ be the simply-connected group with Lie algebra $\mathfrak{g}^{\prime}, \mathfrak{g}^{\prime}=\mathfrak{h}^{\prime} \oplus \mathfrak{p}^{\prime}$ the decomposition into \pm 1 -eigenspaces of $\sigma^{\prime}$ and $\widetilde{K} \subset \widetilde{G}$ the connected subgroup for $\mathfrak{k}^{\prime} \subset \mathfrak{g}^{\prime}$. Let $M$ denote the simply-connected manifold $\widetilde{G} / \widetilde{K}$. We have a projection $\pi: \widetilde{G} \rightarrow M$ given by $\pi(\varphi)=\varphi \widetilde{K}=\varphi\left(x_{0}\right)$ where $x_{0}$ is the coset $\widetilde{K}$. Then the subspace $\mathcal{D}_{x_{0}}=\pi_{*}\left(\mathfrak{p}^{\prime}\right) \subset T_{x_{0}} M$ and the inner product $B^{\prime}$ on $\mathfrak{p}^{\prime}$ projected to $\mathcal{D}_{x_{0}}$ by $\pi_{*}$, denoted by $g_{x_{0}}$, are invariants under $\widetilde{K}$, since $\pi_{*}\left(A d_{k} X\right)=\left(d L_{k}\right)_{x_{0}}\left(\pi_{*} X\right)$ for $k \in \widetilde{K}$ and $X \in \mathfrak{p}^{\prime}$ where $L_{k}: M \rightarrow M, L_{k}(\varphi \widetilde{K})=k \varphi \widetilde{K}$. Hence $\mathcal{D}_{x_{0}}$ and $g_{x_{0}}$ translate respectively to a $\widetilde{G}$-invariant distribution $\mathcal{D}$ on $M$ and to a $\widetilde{G}$-invariant metric $g$ on $\mathcal{D}$. The distribution $\mathcal{D}$ is fat because the linear map $\Theta_{X}$ is onto for all $X \in \mathfrak{p}^{\prime}$. The automorphism $\sigma^{\prime}$ of $\mathfrak{g}^{\prime}$ induces an automorphism $\tau$ of $\widetilde{G}$ such that $\tau_{*}=\sigma^{\prime}$ which in 
turn gives a transformation $\psi: M \rightarrow M$ by the rule $\psi\left(\varphi x_{0}\right)=\tau(\varphi) x_{0}$ for $\varphi \in \widetilde{G}$. Then $\psi$ is an sub-Riemannian isometry of $M$ relative to the sub-Riemannian metric $g$ and $\psi_{*}\left(\pi_{*} X\right)=\pi_{*}\left(\sigma^{\prime} X\right)=-\pi_{*} X$ for $X \in \mathfrak{p}^{\prime}$, so $\psi$ fixes $x_{0}$ and $\psi_{*}$ is -1 on $\mathcal{D}_{x_{0}}$. Thus $\psi$ is the sub-symmetry at $x_{0}$ and $\phi_{\varphi\left(x_{0}\right)}: M \rightarrow M$ defined by $\phi_{\varphi\left(x_{0}\right)}(x)=\varphi \psi \varphi^{-1}(x)$ is the sub-symmetry at $\varphi\left(x_{0}\right)$. Therefore, $M$ is sub-symmetric. In other words,

Theorem 1. Let $\left(\mathfrak{g}^{\prime}, \sigma^{\prime}, \mathfrak{k}^{\prime}, B^{\prime}\right)$ be a fat sub-orthogonal involutive Lie algebra and let $\mathfrak{g} /=\mathfrak{h}^{\prime} \oplus \mathfrak{p}^{\prime}$ denote the decomposition of $\mathfrak{g}^{\prime}$ into the \pm 1 -eigenspaces of the involution $s^{\prime}$. Let $\widetilde{K} \subset \widetilde{H} \subset \widetilde{G}$ be the corresponding connected Lie subgroups for the Lie subalgebras $\mathfrak{k}^{\prime} \subset \mathfrak{g}^{\prime}$ and $\mathfrak{h}^{\prime} \subset \mathfrak{g}^{\prime}$, respectively. Then the total space of the fibration $\widetilde{H} / \widetilde{K} \rightarrow \widetilde{G} / \widetilde{K} \rightarrow \widetilde{G} / \widetilde{H}$ is a fat sub-symmetric space equipped with the $\widetilde{G}$-invariant left-structure determined at the identity coset $\widetilde{K}$ by $\mathfrak{p}^{\prime}$ and $B^{\prime}$.

It follows from the above that the fat sub-OIL algebra $(\mathfrak{g}, \sigma, \mathfrak{k}, B)$ associated to the sub-symmetric space $M$ constructed as above contains $\left(\mathfrak{g}^{\prime}, \sigma^{\prime}, \mathfrak{k}^{\prime}, B^{\prime}\right)$ as a fat sub-OIL subalgebra, that is, $\mathfrak{g}^{\prime}$ is a subalgebra of $\mathfrak{g}, \sigma^{\prime}$ is the restriction of $\sigma, \mathfrak{k}^{\prime}$ is a subalgebra of $\mathfrak{k}$ and $B^{\prime}=B$. Moreover, in general the natural left action of $\widetilde{G}$ on $M$ is not effective; there is a discrete kernel $\widetilde{Z}$. Consequently, $M=G_{0} / K_{0}$ where $G_{0}=\widetilde{G} / \widetilde{Z}$ and $K_{0}=\widetilde{K} / \widetilde{Z}$ still have $\mathfrak{g}^{\prime}$ and $\mathfrak{k}^{\prime}$ as their Lie algebras.

The point here is that given two fat sub-OIL algebra such that one is a fat subOIL subalgebra of the other, the corresponding simply-connected fat sub-symmetric spaces are isometric. Taking this into account, we may assume that $[\mathfrak{p}, \mathfrak{p}]=\mathfrak{h}$ since $[\mathfrak{p}, \mathfrak{p}]+\mathfrak{p}$ is a subalgebra of $\mathfrak{g}$ and we can pass to a fat sub-OIL subalgebra if necessary.

\subsection{A structure theorem}

Let $\beta$ is the Killing form of $\mathfrak{g}$. As Lemma 8.2.1 in [19], we also obtain the following Lemma.

Lemma 1. Let $(\mathfrak{g}, \sigma, \mathfrak{p}, \mathfrak{k}, B)$ be a sub-OIL algebra (recall that we assume that $\left.\mathfrak{p}=\mathfrak{g}^{-}\right)$. Then we can extend the inner product $B$ defined on $\mathfrak{p}$ to an ad $d_{\mathfrak{k}}$-invariant inner product on $\mathfrak{g}$ and we have

- $\beta(\mathfrak{h}, \mathfrak{p})=B(\mathfrak{h}, \mathfrak{p})=0$.

- $\beta_{\left.\right|_{\mathfrak{k}}}$ is negative definite.

Proof. The existence of $B$ and the first property are obvious. To show $\beta_{\left.\right|_{\mathfrak{k}}}$ is negative, observe that $\operatorname{ad}(X)$ is skew-symmetric with respect to $\beta$, so $\operatorname{ad}(X)$ has purely imaginary eigenvalues for all $X \in \mathfrak{k}$ and therefore $\beta(X, X) \leq 0$. If $\beta(X, X)=0$ then all the eigenvalues of $a d_{X}$ would be null and therefore $\operatorname{ad}(X)=0$ and $X$ would be in the centralizer of $\mathfrak{p}$. But we supposed that the adjoint action of $\mathfrak{k}$ on $\mathfrak{p}$ is effective, therefore $X=0$. 
Proposition 1. Let $(\mathfrak{g}, \sigma, \mathfrak{k}, B)$ be a solvable sub-OIL algebra, then $[\mathfrak{g}, \mathfrak{g}] \cap \mathfrak{k}=$ $\{0\}$.

Proof. As $\mathfrak{g}$ is solvable, $\beta(\mathfrak{g},[\mathfrak{g}, \mathfrak{g}])=0$ (Lie's theorem). If $[\mathfrak{g}, \mathfrak{g}] \cap \mathfrak{k} \neq\{0\}$ then there would be an element $k \in \mathfrak{k}$ such that $\beta(k, k)=0$. But $\beta$ is negative definite on $\mathfrak{k}$ and we obtain a contradiction.

As a consequence we obtain the following Corollary.

Corollary 1. Let $(\mathfrak{g}, \sigma, \mathfrak{k}, B)$ be a sub-OIL algebra satisfying $[\mathfrak{p}, \mathfrak{p}]=\mathfrak{h}$. If $\mathfrak{g}$ is solvable then $\mathfrak{k}=0$.

A more precise description of a sub-OIL algebra is obtained by identifying first the maximal solvable ideal in the Lie algebra. We recall now Theorem 5.1 in [14]. Let $(\mathfrak{g}, \sigma)$ be an IL-algebra (Involutive algebras as in [14]) and $\mathfrak{r} \subset \mathfrak{g}$ be its radical, its largest solvable ideal. If $\mathfrak{s} \neq 0$ is an $\operatorname{ad}_{\mathfrak{k}^{-}}, \sigma$ - invariant Levi subalgebra of $\mathfrak{g}$ (cf. [14]), write $\mathfrak{h}_{r}=\mathfrak{h} \cap \mathfrak{r}, \mathfrak{h}_{s}=\mathfrak{h} \cap \mathfrak{s}, \mathfrak{p}_{r}=\mathfrak{p} \cap \mathfrak{r}$ and $\mathfrak{p}_{s}=\mathfrak{p} \cap \mathfrak{s}$. Then $\mathfrak{r}=\mathfrak{h}_{r}+\mathfrak{p}_{r}, \mathfrak{s}=\mathfrak{h}_{s}+\mathfrak{p}_{s}$ and $\mathfrak{g}=\mathfrak{r}+\mathfrak{s}$ is a semidirect sum of IL-algebras.

Theorem 2. The exact sequence of IL-algebras

$$
0 \longrightarrow\left(\mathfrak{r}, \sigma_{\mid \mathfrak{r}}\right) \longrightarrow(\mathfrak{g}, \sigma) \longrightarrow\left(\mathfrak{g} / \mathfrak{r}, \sigma^{*}\right) \longrightarrow 0
$$

where $\sigma^{*}$ is induced by $\sigma$ on the projection, splits.

In the case $\mathfrak{g}$ has a structure of sub-OIL algebra, we obtain a refined theorem.

Theorem 3. Suppose $(\mathfrak{g}, \sigma, \mathfrak{h}, \mathfrak{k}, B)$ is a sub-OIL fat algebra. Then the sequence

$$
0 \longrightarrow\left(\mathfrak{r}, \sigma_{\mid \mathfrak{r}}\right) \longrightarrow(\mathfrak{g}, \sigma) \longrightarrow\left(\mathfrak{g} / \mathfrak{r}, \sigma^{*}\right) \longrightarrow 0
$$

where $\sigma^{*}$ is induced by $\sigma$ on the projection, splits. Moreover, with notations as above, $\mathfrak{r} \cap \mathfrak{k}=\{0\},\left[\mathfrak{p}, \mathfrak{p}_{r}\right]=\mathfrak{h}_{r},\left[\mathfrak{p}_{s}, \mathfrak{p}_{s}\right]=\mathfrak{h}_{s}$ and $\mathfrak{h}_{r}$ is isomorphic to $\mathfrak{h} / \mathfrak{k}$.

Proof. The proof is an immediate consequence of the structure theorem for involutive algebras and the following Lemma.

Lemma 2. Let $(\mathfrak{g}, \sigma, \mathfrak{k}, B)$ be a sub-OIL algebra such that $[\mathfrak{p}, \mathfrak{p}]=\mathfrak{h}$ and let $\mathfrak{r}$ be the radical of $\mathfrak{g}$, then $\mathfrak{r} \cap \mathfrak{k}=\{0\}$.

Proof. Recall $\beta(\mathfrak{r},[\mathfrak{g}, \mathfrak{g}])=0$ (Lie's theorem). If $\mathfrak{r} \cap \mathfrak{k} \neq\{0\}$ then there would be an element $k \in \mathfrak{r}$ such that $\beta(k, k)=0$. But $\beta$ is negative definite on $\mathfrak{k}$ and we obtain a contradiction.

Lemma 3. Let $(\mathfrak{g}, \sigma, \mathfrak{k}, B)$ be a sub-OIL algebra such that $[\mathfrak{p}, \mathfrak{p}]=\mathfrak{h}$. Then $\left[\mathfrak{p}, \mathfrak{p}_{r}\right]=\mathfrak{h}_{r}$ and $\left[\mathfrak{p}_{s}, \mathfrak{p}_{s}\right]=\mathfrak{h}_{s}$. If the algebra is fat then $\mathfrak{h}_{r}$ is isomorphic to $\mathfrak{h} / \mathfrak{k}$. 
Proof. The fact that $\mathfrak{r}$ is an ideal implies that $\left[\mathfrak{p}, \mathfrak{p}_{r}\right] \subset \mathfrak{h}_{r}$ and then both equalities follow from the fact that $[\mathfrak{p}, \mathfrak{p}]=\mathfrak{h}$ as $\mathfrak{p}=\mathfrak{p}_{r} \oplus \mathfrak{p}_{s}$. If the algebra is fat then, on one hand, $\left(\left[\mathfrak{p}, \mathfrak{p}_{r}\right]+\mathfrak{k}\right) / \mathfrak{k}=\mathfrak{h} / \mathfrak{k}$ and on the other hand $\left(\left[\mathfrak{p}, \mathfrak{p}_{r}\right]+\mathfrak{k}\right) / \mathfrak{k}=\left(\mathfrak{h}_{r}+\mathfrak{k}\right) / \mathfrak{k}$ with $\mathfrak{h}_{r} \cap \mathfrak{k}=\{0\}$. This completes the proof.

\subsection{Nilpotent algebras}

Theorem 4. All fat nilpotent sub-OIL algebras are 2-step nilpotent and are obtained as in Example 1.

Proof. By Corollary 1 we know that $\mathfrak{k}=0$. Let the sub-OIL algebra be $(\mathfrak{g}=$ $\mathfrak{p} \oplus \mathfrak{h}, s, \mathfrak{k}=0, B)$. The theorem follows from the following two Lemmas.

Lemma 4. Let $\mathfrak{g}=\mathfrak{p} \oplus \mathfrak{h}$ be a nilpotent Lie algebra with $\mathfrak{h}$ a sub-algebra such that $[\mathfrak{p}, \mathfrak{p}]=\mathfrak{h}$ and $[\mathfrak{p}, \mathfrak{h}] \subset \mathfrak{p}$. Suppose moreover that $i_{X} \circ[\cdot, \cdot]: \mathfrak{p} \rightarrow \mathfrak{h}$ is surjective for every non-zero $X \in \mathfrak{p}$. Then $[\mathfrak{h}, \mathfrak{p}]=0$.

Proof. Observe that, if the Lie algebra is nilpotent and fat, then the last nontrivial ideal in the lower central series will be contained in $\mathfrak{h}$. Denote by $\mathfrak{z}$ that ideal. We show that $[\mathfrak{h}, \mathfrak{p}]=0$ by induction on the dimension of $h$.

If $h$ is one dimensional (or 0 -dimensional), it follows that $\mathfrak{z}=\mathfrak{h}$ coincides with the center of $\mathfrak{g}$ and therefore $[\mathfrak{h}, \mathfrak{p}]=0$.

Now suppose the lemma true in the case $\operatorname{dim} \mathfrak{h}<k$. If the dimension of $\mathfrak{h}$ is $k$ consider the fat nilpotent algebra $\mathfrak{g} / \mathfrak{z}=\mathfrak{p} \oplus \mathfrak{h} / \mathfrak{z}$. We apply the induction hypothesis to conclude that $[\mathfrak{p}, \mathfrak{h} / \mathfrak{z}]=0$ and this equality implies immediately that $[\mathfrak{h}, \mathfrak{p}]=0$.

Lemma 5. Let $\mathfrak{g}=\mathfrak{p} \oplus \mathfrak{h}$ be a Lie algebra with $[\mathfrak{p}, \mathfrak{p}]=\mathfrak{h}$ and $[\mathfrak{p}, \mathfrak{h}]=0$. Then $[\mathfrak{h}, \mathfrak{h}]=0$.

Proof. We may write elements in $\mathfrak{h}$ as $h_{i}=\left[X_{i}, Y_{i}\right], i=1,2$. We obtain therefore

$$
\left[h_{1}, h_{2}\right]=\left[\left[X_{1}, Y_{1}\right],\left[X_{2}, Y_{2}\right]\right]=-\left[\left[Y_{1},\left[X_{2}, Y_{2}\right]\right], X_{1}\right]-\left[\left[\left[X_{2}, Y_{2}\right], X_{1}\right], Y_{1}\right] .
$$

But $[\mathfrak{h}, \mathfrak{p}]=0$, so that the two terms on the right are null as $\left[Y_{1},\left[X_{2}, Y_{2}\right]\right]=\left[\left[X_{2}, Y_{2}\right]\right.$, $\left.X_{1}\right]=0$. This concludes the proof.

To understand 2-step nilpotent algebras it is useful to introduce the following operator.

Definition 6. ([13], [15]) Let $\mathfrak{n}$ be a two-step nilpotent Lie algebra and $\langle\cdot, \cdot\rangle$ be an inner product on $\mathfrak{n}$. Denote by $\mathfrak{z}$ the center of $\mathfrak{n}$ and let $\mathfrak{n}=\mathfrak{z} \oplus \mathfrak{z}^{\perp}$ be the orthogonal decomposition. We can associate to the pair $(\mathfrak{n},<\cdot, \cdot>)$ the linear map

$$
\begin{aligned}
J: \mathfrak{z} & \rightarrow s o\left(\mathfrak{z}^{\perp},<\cdot, \cdot>\left.\right|_{\mathfrak{z}^{\perp}}\right) \\
Z & \mapsto J_{Z}: \mathfrak{z}^{\perp} \rightarrow \mathfrak{z}^{\perp}
\end{aligned}
$$


defined by

$$
<J_{Z} X, Y>\left.\right|_{\mathfrak{z}^{\perp}}=<Z,[X, Y]>\left.\right|_{\mathfrak{z}}, \quad \forall X, Y \in \mathfrak{z}^{\perp}, \forall Z \in \mathfrak{z} .
$$

Conversely, given two real vector spaces $\mathfrak{g}$ and $V$ equipped with the inner products, $\langle\cdot, \cdot\rangle_{\mathfrak{g}}$ and $\left.<\cdot, \cdot\right\rangle_{V}$ respectively, and a linear map $\left.F: \mathfrak{g} \rightarrow s o(V,<\cdot, \cdot\rangle_{V}\right)$. We can define a two-step nilpotent Lie algebra $\mathfrak{n}=\mathfrak{g} \oplus V$ with the Lie bracket given by

$$
\left\{\begin{array}{l}
{[\mathfrak{g}, \mathfrak{g}]=[\mathfrak{g}, V]=0, \quad[V, V] \subset \mathfrak{g},} \\
<[X, Y], Z>_{\mathfrak{g}}=<F(Z) X, Y>_{V}, \quad \forall Z \in \mathfrak{g}, \quad \forall X, Y \in V,
\end{array}\right.
$$

and we can also endow $\mathfrak{n}$ with the inner product $<\cdot, \cdot>$ defined by

$$
<\cdot, \cdot>\left|\mathfrak{g}=<\cdot, \cdot>_{\mathfrak{g}}, \quad<\cdot, \cdot\right|_{V}=<\cdot, \cdot>_{V}, \quad<\mathfrak{g}, V>=0
$$

Definition 7. ([8]) A two-step nilpotent Lie algebra $\mathfrak{n}$ is called fat (also nonsingular, or regular) if $a d_{X}: \mathfrak{n} \rightarrow \mathfrak{z}$ is surjective for all $X \in(\mathfrak{n} \backslash \mathfrak{z})$, where $\mathfrak{z}$ denotes the center of $\mathfrak{n}$.

Proposition 2. ([8], [15]) Let $\mathfrak{n}$ be a two-step nilpotent Lie algebra equipped with an inner product $\langle\cdot, \cdot>$ and denote by $\mathfrak{z}$ the center of $\mathfrak{n}$. Then $[\mathfrak{n}, \mathfrak{n}]=\mathfrak{z}$ if and only if the linear map $J$ associated to the pair $(\mathfrak{n},<\cdot, \cdot\rangle)$ defined as in $(2)$ is such that $J_{Z}$ is not identically null, for all nonzero $Z \in \mathfrak{z}$.

Proof. To show the equivalence we argue by contradiction in both directions. If there exists $Z$ nonzero $\in \mathfrak{z}$, such that $J_{Z}$ is identically null, then $\left\langle J_{Z} X, Y\right\rangle=<$ $[X, Y], Z>=0, \forall X, Y \in \mathfrak{z}^{\perp}$, then $[\mathfrak{n}, \mathfrak{n}]$ cannot be equal to $\mathfrak{z}$ otherwise there will be $X_{1}, Y_{1} \in \mathfrak{z}$ such that $\left[X_{1}, Y_{1}\right]=Z$ and consequently $Z$ would be zero. Conversely, if $[\mathfrak{n}, \mathfrak{n}] \neq \mathfrak{z}$, then there exists $h \in \mathfrak{z}$ such that $h$ is not in $[\mathfrak{n}, \mathfrak{n}]$, since, from the two-step nilpotent hypothesis, $[\mathfrak{n}, \mathfrak{n}] \subset \mathfrak{z}$. Now let $[\mathfrak{n}, \mathfrak{n}]^{\perp}$ be the orthogonal complement to $[\mathfrak{n}, \mathfrak{n}]$ in $\mathfrak{z}$. We have two cases to consider: 1) $h \in[\mathfrak{n}, \mathfrak{n}]^{\perp}$ or 2) $h \notin[\mathfrak{n}, \mathfrak{n}]^{\perp}$. In the first case we would have $\left\langle J_{h} X, Y>=<[X, Y], h>=0 \forall X, Y \in \mathfrak{z}^{\perp}\right.$ and consequently $J_{h} \equiv 0$. In the second case $h=h_{1}+h_{2}$ with $h_{1} \in[\mathfrak{n}, \mathfrak{n}]$ and $h_{2}$ nonzero in $[\mathfrak{n}, \mathfrak{n}]^{\perp}$ and we would have $<J_{h_{2}} X, Y>=<[X, Y], h_{2}>=0 \forall X, Y \in \mathfrak{z}^{\perp}$ and consequently $J_{h_{2}} \equiv 0$. So, in both cases we contradict the hypothesis $J_{Z}$ is not identically null, for all nonzero $Z \in \mathfrak{z}$.

Proposition 3. ([8], [16]) Let $\mathfrak{n}$ be a two-step nilpotent Lie algebra equipped with an inner product $\langle\cdot, \cdot\rangle$ and denote by $\mathfrak{z}$ the center of $\mathfrak{n}$. Then $\mathfrak{n}$ is fat if and only if the linear map $J$ associated to the pair $(\mathfrak{n},\langle\cdot, \cdot\rangle)$ defined as in $(2)$ is such that $J_{Z}$ is invertible, for all nonzero $Z \in \mathfrak{z}$. 
Proof. Follows from the equivalence: $\mathfrak{n}$ is fat if and only if $\forall z \neq 0 \in \mathfrak{z}$ the map $(X, Y) \mapsto<[X, Y], Z>$ is a non-degenerate 2 -form on $\mathfrak{z}^{\perp}$.

Definition 8. ([13]) A Lie algebra $\mathfrak{n}$ equipped with an inner product $\langle\cdot, \cdot\rangle$ is said to be of Heisenberg type or H-type, for short, if it is a two-step nilpotent Lie algebra and the linear map $J$ associated to the pair $(\mathfrak{n},\langle\cdot, \cdot\rangle)$ defined as in $(2)$ is such that $J_{Z}^{2}=-<z, z>I, \forall Z \in \mathfrak{z}$, where $\mathfrak{z}$ denotes the center of $\mathfrak{n}$ and I denotes the identity mapping.

Definition 9. ([16]) A two-step nilpotent Lie algebra $\mathfrak{n}$ equipped with an inner product $\langle\cdot, \cdot\rangle$ is said to be of Representation type or Rep-type, for short, if the two following conditions holds:

i) there is a structure $[\cdot, \cdot]$ of compact Lie algebra on the center $\mathfrak{z}$ of $\mathfrak{n}$;

ii) the linear map $J: \mathfrak{z} \rightarrow s o\left(\mathfrak{z}^{\perp},<\cdot, \cdot>\mid \mathfrak{z}^{\perp}\right)$ associated to the pair $(\mathfrak{n},<\cdot, \cdot>)$ defined as in (2) is a representation of $\mathfrak{z}$, i.e.

$$
J_{[Z, W]}=\left[J_{Z}, J_{W}\right], \quad \forall Z, W \in \mathfrak{z}
$$

Example 2. The quaternionic Heisenberg algebra of dimension $4 n+3, \mathfrak{h}^{4 n+3}$, is the 2-step nilpotent Lie algebra defined by the direct sum $\mathfrak{h}^{4 n+3}=\mathfrak{z} \oplus V$, where $\mathfrak{z}$ is a 3-dimensional real vector space with basis $\alpha=\left\{Z_{1}, Z_{2}, Z_{3}\right\}, V$ is a $4 n$-dimensional real vector space with bases $\beta=\left\{X_{1}, Y_{1}, V_{1}, W_{1}, \ldots, X_{n}, Y_{n}, V_{n}, W_{n}\right\}$ and the bracket operation in $\mathfrak{h}^{4 n+3}$ is defined as follows:

$$
\begin{array}{lllll}
{\left[X_{i}, Y_{i}\right]=Z_{1},} & {\left[X_{i}, V_{i}\right]=Z_{2},} & {\left[X_{i}, W_{i}\right]=Z_{3},} & \text { for } & 1 \leq i \leq n, \\
{\left[Y_{i}, X_{i}\right]=-Z_{1},} & {\left[Y_{i}, V_{i}\right]=Z_{3},} & {\left[Y_{i}, W_{i}\right]=-Z_{2},} & \text { for } \quad 1 \leq i \leq n, \\
{\left[V_{i}, X_{i}\right]=-Z_{2},} & {\left[V_{i}, Y_{i}\right]=-Z_{3},} & {\left[V_{i}, W_{i}\right]=Z_{1},} & \text { for } \quad 1 \leq i \leq n, \\
{\left[W_{i}, X_{i}\right]=-Z_{3},} & {\left[W_{i}, Y_{i}\right]=Z_{2},} & {\left[W_{i}, V_{i}\right]=-Z_{1},} & \text { for } & 1 \leq i \leq n,
\end{array}
$$

and all the other brackets are zero.

In the quaternionic Heisenberg algebra case, setting the inner product $\langle\cdot, \cdot\rangle_{\mathfrak{h}}$ on $\mathfrak{h}^{4 n+3}$ that declares $\alpha$ and $\beta$ to be orthonormal basis and $\mathfrak{z}$ and $V$ orthogonal subspaces, the linear map defined in (2) associated to the pair $\left(\mathfrak{h}^{4 n+3},<\cdot, \cdot>_{\mathfrak{h}}\right)$ satisfies:

$$
\begin{aligned}
& J_{Z_{1}}: V \rightarrow V \quad J_{Z_{2}}: V \rightarrow \quad V \quad J_{Z_{3}}: V \rightarrow \quad V \\
& \begin{array}{llll}
X_{i} \rightarrow Y_{i} & X_{i} \rightarrow V_{i} & X_{i} \rightarrow W_{i}
\end{array} \\
& Y_{i} \rightarrow-X_{i} \quad Y_{i} \rightarrow-W_{i} \quad Y_{i} \rightarrow \quad V_{i} \quad(\text { for } \quad 1 \leq i \leq n) \text {. } \\
& V_{i} \rightarrow W_{i} \quad V_{i} \rightarrow-X_{i} \quad V_{i} \rightarrow-Y_{i} \\
& W_{i} \rightarrow-V_{i} \quad W_{i} \rightarrow \quad Y_{i} \quad W_{i} \rightarrow-X_{i}
\end{aligned}
$$


And then,

$$
\begin{aligned}
& J_{Z_{1}} \circ J_{Z_{2}}-J_{Z_{2}} \circ J_{Z_{1}}=2 J_{Z_{3}}, \\
& J_{Z_{1}} \circ J_{Z_{3}}-J_{Z_{3}} \circ J_{Z_{1}}=-2 J_{Z_{2}}, \\
& J_{Z_{2}} \circ J_{Z_{3}}-J_{Z_{3}} \circ J_{Z_{2}}=2 J_{Z_{1}} .
\end{aligned}
$$

So, $J(\mathfrak{z})$ is a Lie subalgebra of $s o\left(V,<\cdot, \cdot>\left.\right|_{V}\right)$.

Example 3. Let $V_{2}^{3}$ be the real vector space of all homogeneous polynomials of degree 3 in two complex variables $u$ and $w$ with complex coefficients, i.e. $V_{2}^{3}=$ $\left\{P(u, w)=a u^{3}+b u^{2} w+c u w^{2}+d w^{3}: a, b, c, d \in \mathbb{C}\right\}$. Setting the inner product $\left.<\cdot, \cdot\right\rangle_{V}$ on $V_{2}^{3}$, that declares $\left\{\frac{1}{\sqrt{3}} u^{3}, i \frac{1}{\sqrt{3}} u^{3}, u^{2} w, i u^{2} w, u w^{2}, i u w^{2}, \frac{1}{\sqrt{3}} w^{3}, i \frac{1}{\sqrt{3}} w^{3}\right\}$ to be an orthonormal basis. Consider the real Lie algebra $s u(2)$ equipped with the inner product such that the basis $\left\{H=\left[\begin{array}{cc}i & 0 \\ 0 & -i\end{array}\right], E_{-}=\left[\begin{array}{cc}0 & -1 \\ 1 & 0\end{array}\right], E_{+}=\left[\begin{array}{cc}0 & -i \\ -i & 0\end{array}\right]\right\}$ is orthonormal. Consider also the linear map

$$
\begin{aligned}
F: s u(2) & \rightarrow s o\left(V_{2}^{3},<\cdot, \cdot>_{V}\right) \\
Z & \mapsto F_{Z}: s o\left(V_{2}^{3}\right) \rightarrow s o\left(V_{2}^{3}\right)
\end{aligned}
$$

induced by the irreducible representation $V_{2}^{3}$ of $S U(2)$ regarded as a real representation, i.e.

$$
\begin{gathered}
F_{Z}(P(u, w))=\left.\frac{d}{d t}\right|_{t=0} P\left(e^{-t Z}\left[\begin{array}{c}
u \\
w
\end{array}\right]=d P_{(u, w)}\left(-Z\left[\begin{array}{c}
u \\
w
\end{array}\right]\right)\right. \\
=-\left[\frac{\partial P}{\partial u}(u, w) \frac{\partial P}{\partial w}(u, w)\right]\left(Z\left[\begin{array}{c}
u \\
w
\end{array}\right]\right) .
\end{gathered}
$$

Then, we can associate to these structures the two-step nilpotent Lie algebra $\mathfrak{n}=s u(2) \oplus V_{2}^{3}$ with the Lie bracket defined as in 3 which can be represented by its structural map $F_{z}$ given in the fixed orthonormal basis by

$$
F_{a H+b E_{-}+c E_{+}}=\left[\begin{array}{cccccccc}
0 & 3 a & -\sqrt{3} b & -\sqrt{3} c & 0 & 0 & 0 & 0 \\
-3 a & 0 & \sqrt{3} c & -\sqrt{3} b & 0 & 0 & 0 & 0 \\
\sqrt{3} b & -\sqrt{3} c & 0 & a & -2 b & -2 c & 0 & 0 \\
\sqrt{3} c & \sqrt{3} b & -a & 0 & 2 c & -2 b & 0 & 0 \\
0 & 0 & 2 b & -2 c & 0 & -a & -\sqrt{3} b-\sqrt{3} c \\
0 & 0 & 2 c & 2 b & a & 0 & \sqrt{3} c & -\sqrt{3} b \\
0 & 0 & 0 & 0 & \sqrt{3} b-\sqrt{3} c & 0 & -3 a \\
0 & 0 & 0 & 0 & \sqrt{3} c & \sqrt{3} b & 3 a & 0
\end{array}\right]
$$

Note that $\operatorname{det} F_{z} \neq 0, \forall z \neq 0 \in s u(2)$ and then, by Proposition $3, \mathfrak{n}$ is fat. 
Remark 3. Denote by $V_{2}^{n}$ the real vector space of all homogeneous polynomials of degree $\mathrm{n}$ in two complex variables $\mathrm{u}$ and $\mathrm{w}$ with complex coefficients, i.e. $V_{2}^{n}=\left\{P(u, w)=a_{n} u^{n}+a_{n-1} u^{n-1} w+\ldots+a_{1} u w^{n-1}+a_{0} w^{n}: a_{i} \in \mathbb{C}, i=0,1, \ldots, n\right\}$. Setting the inner product $\langle\cdot, \cdot\rangle_{V}$ on $V_{2}^{n}$ such that

$$
\left\{X_{0}, i X_{0}, X_{1}, i X_{1}, \ldots, X_{l}, i X_{l}, Y_{l+1}, i Y_{l+1}, \ldots, Y_{n-1}, i Y_{n-1}, Y_{0}, i Y_{0}\right\}
$$

is an orthonormal basis, where $X_{0}=\frac{1}{\sqrt{n}} u^{n}, X_{1}=u^{n-1} w, Y_{0}=\frac{1}{\sqrt{n}} w^{n}, Y_{n-1}=u w^{n-1}$, $X_{k}=\frac{\sqrt{n-1}}{\sqrt{2}} \ldots \frac{\sqrt{n-(k-1)}}{\sqrt{k}} u^{n-k} w^{k}(k=2, \ldots, l), \quad Y_{s}=\frac{\sqrt{n-1}}{\sqrt{2}} \ldots \frac{\sqrt{s+1}}{\sqrt{n-s}} u^{n-s} w^{s}(s=l+1, \ldots$, $n-2)$ and $l=\frac{n}{2}$ if $n$ is even or $l=\frac{n-1}{2}$ if $n$ is odd. Consider the real Lie algebra $s u(2)$ equipped with the inner product such that the basis $\left\{Z_{1}=-H=\left[\begin{array}{cc}-i & 0 \\ 0 & i\end{array}\right], Z_{2}=\right.$ $\left.E_{+}=\left[\begin{array}{cc}0 & -i \\ -i & 0\end{array}\right], Z_{3}=E_{-}=\left[\begin{array}{cc}0 & -1 \\ 1 & 0\end{array}\right]\right\}$ is orthonormal. Consider also the linear map

$$
\begin{aligned}
F: s u(2) & \rightarrow s o\left(V_{2}^{n},<\cdot, \cdot>_{V}\right) \\
Z & \mapsto F_{Z}: s o\left(V_{2}^{n}\right) \rightarrow s o\left(V_{2}^{n}\right)
\end{aligned}
$$

induced by the irreducible representation $V_{2}^{n}$ of $S U(2)$ regarded as a real representation, i.e.

$$
F_{Z}(P(u, w))=\left.\frac{d}{d t}\right|_{t=0} P\left(e^{-t Z}\left[\begin{array}{c}
u \\
w
\end{array}\right]=-\left[\frac{\partial P}{\partial u}(u, w) \frac{\partial P}{\partial w}(u, w)\right]\left(Z\left[\begin{array}{c}
u \\
w
\end{array}\right]\right)\right.
$$

Then, we can associate to this structure the two-step nilpotent Lie algebra $\mathfrak{n}=s u(2) \oplus V_{2}^{n}$ with the Lie bracket defined as in 3 by its structural map $F_{z}$. In particular when $n=1$, the associated two-step nilpotent Lie algebra $\mathfrak{n}=s u(2) \oplus V_{2}^{1}$ is the 7 - dimensional quaternionic Heisenberg algebra.

Example 4. In [1] we find a canonical way of building up an irreducible simplyconnected fat sub-Riemannian symmetric space as a $S p(1)$-fibration over a compact simply-connected quaternionic Wolf space (i.e., over a Riemannian symmetric space equipped with a quaternionic structure for which the holonomy group has quaternionic scalar part). The sub-OIL fat algebra $(\mathfrak{g}, s, \mathfrak{k}, B)$ associated to this class of examples of sub-symmetric spaces of quaternionic type has the following additional properties:

i. the decomposition of $\mathfrak{g}$ into the \pm 1 -eigenspaces of the involution $s$ denoted by $\mathfrak{g}=\mathfrak{h} \oplus \mathfrak{p}$ is such that the +1 -eigenspace is a direct sum of ideals $\mathfrak{h}=\mathfrak{k} \oplus \mathfrak{a}$ with $\mathfrak{a} \cong \mathfrak{s p}(1)$ and $\mathfrak{h}=[\mathfrak{p}, \mathfrak{p}]$; 
ii. there is a $B$-orthonormal basis $\left\{X_{1}, J_{1}\left(X_{1}\right), J_{2}\left(X_{1}\right), J_{3}\left(X_{1}\right), \ldots, X_{n}, J_{1}\left(X_{n}\right)\right.$, $\left.J_{2}\left(X_{n}\right), J_{3}\left(X_{n}\right)\right\}$ of $\mathfrak{p}$ that satisfies:

$$
\begin{aligned}
{\left[X_{k}, J_{1}\left(X_{k}\right)\right] } & \equiv \frac{a}{-4 n-8} \quad \xi_{1} \quad \bmod \mathfrak{k}, \\
{\left[X_{k}, J_{2}\left(X_{k}\right)\right] } & \equiv \frac{a}{-4 n-8} \quad \xi_{2} \quad \bmod \mathfrak{k}, \\
{\left[X_{k}, J_{3}\left(X_{k}\right)\right] } & \equiv \frac{a}{-4 n-8} \quad \xi_{3} \bmod \mathfrak{k}, \\
{\left[J_{1}\left(X_{k}\right), J_{2}\left(X_{k}\right)\right] } & \equiv \frac{a}{-4 n-8} \quad \xi_{3} \bmod \mathfrak{k}, \\
{\left[J_{1}\left(X_{k}\right), J_{3}\left(X_{k}\right)\right] } & \equiv-\frac{a}{-4 n-8} \quad \xi_{2} \quad \bmod \mathfrak{k}, \\
{\left[J_{2}\left(X_{k}\right), J_{3}\left(X_{k}\right)\right] } & \equiv \frac{a}{-4 n-8} \quad \xi_{1} \quad \bmod \mathfrak{k}, \quad \text { for } \quad k=1,2, \ldots, n,
\end{aligned}
$$

and the other brackets are zero modulo $\mathfrak{k}$, where $a$ is a positive scalar and $\gamma=$ $\left\{\xi_{1}, \xi_{2}, \xi_{3}\right\}$ is the basis of $\mathfrak{a}$ satisfying $\left[\xi_{1}, \xi_{2}\right]=2 \xi_{3},\left[\xi_{1}, \xi_{3}\right]=-2 \xi_{2}$, and $\left[\xi_{2}, \xi_{3}\right]=2 \xi_{1}$.

Now, consider the inner product $\langle\cdot, \cdot\rangle$ on $[\mathfrak{p}, \mathfrak{p}] / \mathfrak{k}$ such that $\left\{\frac{a}{-4 n-8} \xi_{1} \bmod \mathfrak{k}\right.$, $\left.\frac{a}{-4 n-8} \xi_{2} \bmod \mathfrak{k}, \frac{a}{-4 n-8} \xi_{3} \bmod \mathfrak{k}\right\}$ is an orthonormal basis of $[\mathfrak{p}, \mathfrak{p}] / \mathfrak{k}$ and the linear map

$$
\begin{aligned}
F:[\mathfrak{p}, \mathfrak{p}] / \mathfrak{k} & \rightarrow s o(\mathfrak{p}, B) \\
Z & \mapsto F_{Z}: \mathfrak{p} \rightarrow \mathfrak{p}
\end{aligned}
$$

defined by setting

$$
B\left(F_{Z} X, Y\right)=<Z, \theta_{X}(Y)>=<Z,[X, Y] \quad \bmod \mathfrak{k}>, \quad \forall X, Y \in \mathfrak{p}, \forall Z \in[\mathfrak{p}, \mathfrak{p}] / \mathfrak{k},
$$

i.e., $F_{Z}(X)=\left(\theta_{X}\right)^{*}(Z)$ where $\left(\theta_{X}\right)^{*}$ denotes the adjoint of the surjective linear map $\Theta_{X}: \mathfrak{p} \rightarrow[\mathfrak{p}, \mathfrak{p}] / \mathfrak{k}, \Theta_{X}(Y)=[X, Y] \bmod \mathfrak{k}$. Then, the two-step nilpotent Lie algebra $\mathfrak{n}=[\mathfrak{p}, \mathfrak{p}] / \mathfrak{k} \oplus \mathfrak{p}$ associated to these structures, with the Lie bracket defined as in 3 by its structural map $F_{z}$ is the quaternionic Heisenberg algebra of dimension $4 n+3$.

\section{Geometric invariants}

In this section we show, by introducing a connection adapted to a fat distribution, that one can interpret fundamental examples as flat spaces with constant torsions. Namely, the quaternionic Heisenberg model, $\mathfrak{h}^{4 n+3}$, described in Example 2 and the model given in Example 3. We also relate the adapted invariants to previous work on nilpotent groups with the viewpoint of Riemannian geometry.

Let $\mathfrak{n}$ be a two-step nilpotent Lie algebra and $\langle\cdot, \cdot\rangle$ be an inner product on $\mathfrak{n}$. Denote by $\mathfrak{z}$ the center of $\mathfrak{n}$ and let $\mathfrak{n}=\mathfrak{z} \oplus \mathfrak{z}^{\perp}$ be the orthogonal decomposition. Denote by $(N,<\cdot, \cdot>)$ a simply real nilpotent Lie group with Lie algebra $\mathfrak{n}$ endowed 
with a left-invariant metric determined by $\langle\cdot, \cdot\rangle$. The Levi-Civita connection, denoted by $\bar{\nabla}$, and the curvature and Ricci tensors of $(N,<\cdot, \cdot>)$ has been computed in [8]. One obtains

$$
\bar{\nabla}_{U} V=\frac{1}{2}\left\{[U, V]-(a d U)^{*}(V)-(a d V)^{*}(U)\right\}
$$

where $U, V$ are elements of $\mathfrak{n}$ regarded as left invariant vector fields on $N$, and $(a d U)^{*}$ and $(a d V)^{*}$ denote the adjoints of $a d U$ and $a d V$. In particular, one has

$$
\left\{\begin{array}{lrl}
\text { i) } & \bar{\nabla}_{X} Y=\frac{1}{2}[X, Y] & \forall X, Y \in \mathfrak{z}^{\perp}, \\
\text { ii) } & \bar{\nabla}_{X} Z=\bar{\nabla}_{Z} X=-\frac{1}{2} J_{Z}(X) & \forall X \in \mathfrak{z}^{\perp}, \forall Z \in \mathfrak{z}, \\
\text { iii) } & \bar{\nabla}_{Z} W=0 & \forall Z, W \in \mathfrak{z} .
\end{array}\right.
$$

A canonical connection adapted to the distribution defined on a sub-Riemannian manifold which has an intrinsic Riemannian extension to the whole manifold was constructed in [1]. To be more precise, let $(M,<\cdot, \cdot>)$ be a smooth Riemannian manifold and $\mathcal{D}$ a smooth distribution on $M$. Let $\mathcal{D}^{\perp}$ be the $\langle\cdot, \cdot>$-orthonormal complement of $\mathcal{D}$. Finally, let $\underline{T M}, \underline{\mathcal{D}}$ and $\underline{\mathcal{D}}^{\perp}$ denote respectively the set of sections of $T M, \mathcal{D}$ and $\mathcal{D}^{\perp}$. Then

Theorem 5. ([1]) There exists a unique connection $\nabla: \underline{T M} \rightarrow \underline{T M}^{*} \otimes \underline{T M}$, called the adapted connection, and unique tensors $\tau: \mathcal{D}^{\perp} \otimes \mathcal{D} \rightarrow \overline{\mathcal{D}}$ and $\overline{\sigma: \mathcal{D}} \otimes \overline{\mathcal{D}^{\perp} \rightarrow}$ $\mathcal{D}^{\perp}$ which are symmetric in the second variable, called the sub-torsions, with the following properties ( $T$ is the torsion tensor of the connection):

a. $\quad \nabla_{U}: \underline{\mathcal{D}} \rightarrow \underline{\mathcal{D}}, \quad \nabla_{U}: \underline{\mathcal{D}}^{\perp} \rightarrow \underline{\mathcal{D}}^{\perp} ;$

b. $\nabla<\cdot, \cdot>=0$;

c. $T(\xi, X)=\tau_{\xi}(X)+\sigma_{X}(\xi)$,

$T(X, Y)=-[X, Y]^{\perp}$, $T(\xi, \eta)=-[\xi, \eta]^{\top}$

for $X, Y \in \underline{\mathcal{D}} ; \xi, \eta \in \underline{\mathcal{D}}^{\perp}$ and $U \in \underline{T M}$, where $[X, Y]^{\perp}$ denotes the projection of $[X, Y]$ onto $\mathcal{D}^{\perp}$ and $[\xi, \eta]^{\top}$ denotes the projection of $[\xi, \eta]$ onto $\mathcal{D}$.

We compute the adapted connection for the Riemannian manifold $(N,<\cdot, \cdot>)$ and it turns out that the adapted connection and the sub-torsions are identically null. The only torsion which is not identically zero is $T(X, Y)=-[X, Y]$, for $X$, $Y \in \mathfrak{z}^{\perp}$.

We relate the adapted connection to the invariants of the Levi-Civita connection as computed in [8]. For that, we recall that the map $J_{Z}: \mathfrak{z}^{\perp} \rightarrow \mathfrak{z}^{\perp}$ is the unique skew-symmetric linear map such that $\left\langle J_{Z} X, Y>\left.\right|_{\mathfrak{z}^{\perp}}=\left.\langle Z,[X, Y]\rangle\right|_{\mathfrak{z}}\right.$, 
$\forall X, Y \in \mathfrak{z}^{\perp}, \forall Z \in \mathfrak{z}$. Let $\operatorname{End}(\mathfrak{z})$ and $\operatorname{End}\left(\mathfrak{z}^{\perp}\right)$ be respectively the assembly of all endomorphism of $\mathfrak{z}$ and $\mathfrak{z}^{\perp}$, and denote by Ric the Ricci tensor associated to the LeviCivita connection. Define the bilinear map $\bar{b}: \mathfrak{z}^{\perp} \times \mathfrak{z}^{\perp} \rightarrow \operatorname{End}(\mathfrak{z}), \bar{b}(\mathrm{X}, \mathrm{Y})=\bar{b}_{\mathrm{XY}}: \mathfrak{z} \rightarrow \mathfrak{z}$ given by

$$
\bar{b}_{\mathrm{XY}}(Z)=\left[J_{Z}(X), Y\right]=T\left(Y, J_{Z}(X)\right), \quad \forall X, Y \in \mathfrak{z}^{\perp}, \forall Z \in \mathfrak{z} .
$$

Then, the linear map

$$
\hat{b}=\operatorname{trace} \bar{b}=\sum_{i=1}^{m} \bar{b}_{e_{i} e_{i}}: \mathfrak{z} \longrightarrow \mathfrak{z}
$$

where $\beta=\left\{e_{1}, e_{2}, \ldots, e_{m}\right\}$ is an orthonormal basis of $\mathfrak{z}^{\perp}$, does not depend on $\beta$ and it is a symmetric linear map. This fact allows us to define the invariants $\lambda_{1}, \ldots, \lambda_{d}$ eigenvalues of $\hat{b}$. Note that, in the basis $\beta$ of $\mathfrak{z}^{\perp}$,

$$
\operatorname{trace}\left(J_{Z} \circ J_{W}\right)=\sum_{i=1}^{m}<J_{Z} \circ J_{W}\left(e_{i}\right), e_{i}>\left.\right|_{\mathfrak{z}^{\perp}}=<\sum_{i=1}^{m} \bar{b}_{e_{i} e_{i}}(Z), W>\left.\right|_{\mathfrak{z}} .
$$

And, from [8], we have that $\operatorname{trace}\left(J_{Z} \circ J_{W}\right)=-4 \operatorname{Ric}(Z, W), \forall Z, W \in \mathfrak{z}$. So, $\hat{b}: \mathfrak{z} \rightarrow \mathfrak{z}$ defined as in 4 is the unique positive semidefinite symmetric linear map such that $-4 \operatorname{Ric}(Z, W)=<\hat{b}(Z), W>\left.\right|_{\mathfrak{z}}, \forall Z, W \in \mathfrak{z}$.

Also, the bilinear map

$$
\begin{aligned}
& A: \mathfrak{z} \times \mathfrak{z} \longrightarrow \operatorname{End}\left(\mathfrak{z}^{\perp}\right) \\
& (Z, W) \longmapsto J_{Z^{\circ} J_{W}}
\end{aligned}
$$

induces the symmetric linear map

$$
\hat{A}=\operatorname{trace} A=\sum_{\alpha=1}^{d} A\left(E_{\alpha}, E_{\alpha}\right)=\sum_{\alpha=1}^{d}\left(J_{E_{\alpha}}\right)^{2}: \mathfrak{z}^{\perp} \longrightarrow \mathfrak{z}^{\perp}
$$

where $\left\{E_{1}, \ldots, E_{d}\right\}$ is a orthonormal basis of $\mathfrak{z}$, and provide us the invariants $\rho_{i}(i=$ $1, \ldots, m)$ eigenvalues of $\hat{A}$. And, again from [8], we have that $<\frac{1}{2} \sum_{\alpha=1}^{d}\left(J_{E_{\alpha}}\right)^{2}(X)$, $Y>\left.\right|_{\mathfrak{z}^{\perp}}=\operatorname{Ric}(X, Y), \forall X, Y \in \mathfrak{z}^{\perp}$. So, $\hat{A}: \mathfrak{z}^{\perp} \rightarrow \mathfrak{z}^{\perp}$ defined as in 5 is the unique negative definite symmetric linear map such that $2 \operatorname{Ric}(X, Y)=<\hat{A}(X), Y>\left.\right|_{\mathfrak{z}^{\perp}}, \forall X, Y \in \mathfrak{z}^{\perp}$.

Remark 4. We calculate the geometrical invariants for the quaternionic Heisenberg model, $\mathfrak{h}^{4 n+3}$, described in Example 2 and also for the model given in Example 3. We find $\rho_{1}=\rho_{2}=\ldots=\rho_{4 n}=-3$ and $\lambda_{1}=\lambda_{2}=\lambda_{3}=-4 n$ for the Example 2 . For the Example 3, we find $\rho_{1}=\rho_{2}=\ldots=\rho_{8}=-15$ and $\lambda_{1}=\lambda_{2}=\lambda_{3}=-40$. For the $\mathfrak{n}=s u(2) \oplus V_{2}^{n}$ example with the inner product defined as in Remark 3 , we find $\rho_{1}=\rho_{2}=\ldots=\rho_{2 n+2}=-n^{2}-2 n$ and $\lambda_{1}=\lambda_{2}=\lambda_{3}=-\frac{2}{3}\left(n^{3}+3 n^{2}+2 n\right)$. 


\section{Naturally reductive Nilpotent algebras}

It is important to remark that the complete classification of two-step nilpotent fat algebras in dimension greater than 7 and 3-dimensional center (the 7-dimensional case will be studied in the next section) is difficult. As an example, as shown in [16], there exists explicit examples of continuous families of non-isomorphic fat two-step algebras in higher dimensions.

The classification, however, is possible if we restrict the class of nilpotent groups. In this section we present the classification of fat two-step nilpotent Lie algebras of Rep-type with 3-dimensional center, Corollary 2 and Corollary 3. Using that classification and the classification of H-type Lie algebras given in [3] we found out that the quaternionic Heisenberg algebra is the only algebra with 3-dimensional center figuring in both classes.

We start with a definition of naturally reductive Lie algebra which is closely related to a special class of Rep-type Lie algebras. It provides an interesting class of homogeneous spaces which generalize the class of symmetric spaces.

Definition 10. ([12]) The homogeneous Riemannian manifold $(M, g)$ is said to be naturally reductive if there exists:

1) a Lie group $\mathrm{G}$ acting transitively and effectively on the left on $M$ as group of isometries,

2 ) a vector space $\mathfrak{m}$ complement to $\mathfrak{k}$ in $\mathfrak{g}$ such that $A d(K) \mathfrak{m} \subseteq \mathfrak{m}$, where $K$ denotes the isotropy subgroup of $G$ at some point $p \in M$, $\mathfrak{g}$ and $\mathfrak{k}$ denote the Lie algebras of $G$ and $K$,

satisfying the property

$$
<[X, Y]_{\mathfrak{m}}, Z>+<Y,[X, Z]_{\mathfrak{m}}>=0, \quad \forall X, Y, Z \in \mathfrak{m},
$$

where $[X, Y] \mathfrak{m}$ denotes the projection of $[X, Y]$ on $\mathfrak{m}$ with respect to the decomposition $\mathfrak{g}=\mathfrak{k} \oplus \mathfrak{m}$ and $\langle\cdot, \cdot\rangle$ denotes the inner product on $\mathfrak{m}$ induced by the metric $g$.

Proposition 4. ([12], [15]) Let $\mathfrak{n}$ be a two-step nilpotent Lie algebra with center $\mathfrak{z}$ such that $[\mathfrak{n}, \mathfrak{n}]=\mathfrak{z}$ and $\langle\cdot, \cdot>$ be an inner product on $\mathfrak{n}$. Denote by $(N,<\cdot, \cdot>)$ a simply connected real nilpotent Lie group with Lie algebra $\mathfrak{n}$ endowed with a leftinvariant metric determined by $<\cdot, \cdot\rangle$. Then,$(N,<\cdot, \cdot\rangle)$ is naturally reductive if and only if there exists a compact Lie algebra structure on $\mathfrak{z}$ given by a bilinear map $\tau: \mathfrak{z} \times \mathfrak{z} \rightarrow \mathfrak{z}$ with the property $\tau(x, \cdot) \in \operatorname{so}(\mathfrak{z},<\cdot, \cdot>\mid \mathfrak{z})$ such that $J: \mathfrak{z} \rightarrow \operatorname{so}\left(\mathfrak{z}^{\perp},<\cdot, \cdot>\left.\right|_{\mathfrak{z}^{\perp}}\right)$ becomes a real faithful representation of the Lie algebra $(\mathfrak{z}, \tau)$ on $\mathfrak{z}^{\perp}$ without trivial subrepresentations, $i$. e. $\bigcap_{z \in \mathfrak{z}}$ ker $J_{z}=0$.

Corollary 2. Let $\mathfrak{n}$ be a fat two-step nilpotent Lie algebra with center $\mathfrak{z}$ and $<\cdot, \cdot>$ be an inner product on $\mathfrak{n}$. Denote by $(N,<\cdot, \cdot>)$ a simply connected real nilpotent Lie group with Lie algebra $\mathfrak{n}$ endowed with a left-invariant metric determined by 
$<\cdot, \cdot>$. Then, $(N,<\cdot, \cdot>)$ is naturally reductive if and only if there exists a compact Lie algebra structure $\tau$ on $\mathfrak{z}$ such that $(\mathfrak{z}, \tau) \cong s u(2)$ and $J: \mathfrak{z} \rightarrow \operatorname{so}\left(\mathfrak{z}^{\perp},\left\langle\cdot, \cdot>\left.\right|_{\mathfrak{z}^{\perp}}\right)\right.$ is a real faithful representation of $s u(2)$ without trivial subrepresentations.

Proof. First observe that the two-step nilpotent and fat hypotheses imply via Proposition 2 and Proposition 3 that $[\mathfrak{n}, \mathfrak{n}]=\mathfrak{z}$. Next note that, using Proposition 4 , it only remains to proof the isomorphism $(\mathfrak{z}, \tau) \cong s u(2)$. To see this we are first going to demonstrate that $\operatorname{rank}(\mathfrak{z})=1$. Suppose to the contrary, i. e. $\operatorname{rank}(\mathfrak{z})>1$. Since $J: \mathfrak{z} \rightarrow s o\left(\mathfrak{z}^{\perp},<\cdot, \cdot>\left.\right|_{\mathfrak{z}^{\perp}}\right)$ is a faithful representation, it follows that $(\mathfrak{z}, \tau) \cong J(\mathfrak{z})$, so $\operatorname{rank}(J(\mathfrak{z}))=1$. Consequently, there exists $T$ and $F$, linearly independent, in $J(\mathfrak{z})$ that commute. Since $T$ and $F$ are skew-symmetric, invertible and commute, there exits $W$ a proper subspace of $\mathfrak{z}^{\perp}$ invariant by $T$ and $F$, and then $\left.T\right|_{W}=\left.\lambda F\right|_{W}$ for some real $\lambda \neq 0$. So, the map $T-\lambda F \neq 0$, since $T$ and $F$ are linearly independent, but $\left.(T-\lambda F)\right|_{W} \equiv 0$. This fact is a contradiction since $T-\lambda F$ is in $J(\mathfrak{z})$ and the elements of $J(\mathfrak{z})$ are invertible operators. So, we have $\mathfrak{z}$ compact with $\operatorname{rank}(\mathfrak{z})=1$, then by [[6], Theorem 1.5 and its Corollary] it follows that $(\mathfrak{z}, \tau) \cong s u(2)$.

Corollary 3. Let $\mathfrak{n}$ be a fat two-step nilpotent Lie algebra (equipped with an inner product) which is of Rep-type and with three dimensional center $\mathfrak{z}$. If the map $J$ defined as in (2) is an irreducible representation of $\mathfrak{z}$ then it is isomorphic to the irreducible representation $V_{2}^{k}$ of $s u(2)$ with $k=\frac{1}{2} \operatorname{dim}_{\mathfrak{z}}{ }^{\perp}-1$, regarded as a real representation, as in Remark (3). In the reducible case, by the complete reducibility property of su(2), it is isomorphic to a direct sum of a finite number of the irreducible representations $V_{2}^{n}$ of $s u(2)$ with $n$ odd.

\section{Classification in dimension 7}

In this section we prove that, in dimension 7 , the only nilpotent sub-OIL fat algebra with distribution of codimension 3 is the quaternionic Heisenberg algebra of Example 2 (see Corollary 4). This is the analog of the fact that the only nilpotent contact sub-symmetric spaces are the Heisenberg groups. It is a fundamental piece for a full classification of sub-symmetric spaces of dimension 7 which we hope to achieve.

In general, it is not true that a fat two-step nilpotent Lie algebra $\mathfrak{n}$ equipped with an inner product is of Rep-type, even under the assumption that the center $z$ of $\mathfrak{n}$ has dimension three; see some counterexamples in [16]. But, by [[7], Lemma 3] the assertion holds if $\operatorname{dim} \mathfrak{n}=7$ and $\operatorname{dim} \mathfrak{z}=3$. For the sake of completeness we give a proof following [7].

Lemma 6. Let $V$ and $W$ be real vector space of finite dimensions, and let $[\cdot, \cdot]: V \times V \rightarrow W$ be a skew-symmetric bilinear map. Then, $i_{X} \circ[\cdot, \cdot]: V \times V \rightarrow W$ is 
onto for all nonzero $X \in V$ if and only if, for any nonzero functional $f \in W^{*}$, the composite $(f \circ[\cdot, \cdot])$ is a non-degenerate skew-symmetric bilinear form.

Proof. To show the equivalence we argue by contradiction in both directions. In fact, if there exists $f$ nonzero in $W^{*}$, such that the composite $(f \circ[\cdot, \cdot])$ is a degenerate skew-symmetric bilinear form, then there exists a nonzero $X \in V$ such that $(f \circ[X, Y])=0 \forall Y \in V$ and, consequently, for this $X$ the map $i_{X} \circ[\cdot, \cdot]$ cannot be surjective. Conversely, if there exists a non-trivial $X \in V$ such that $i_{X} \circ[\cdot, \cdot]: V \times V \rightarrow$ $W$ is not onto, then there exists a non-trivial $Y \in W$ such that $Y$ is not in the image of $i_{X} \circ[\cdot, \cdot]$. Then complete $Y$ to a basis $\beta$ of $W$ and define a nonzero functional $f \in W^{*}$ by setting $f(Y) \neq 0$ and $f \equiv 0$ for other elements of the basis $\beta$. So, for these $f \in W^{*}$ we have that $(f \circ[\cdot, \cdot]) \equiv 0$ or, equivalently, that $(f \circ[\cdot, \cdot])$ is a degenerate skew-symmetric bilinear form.

Lemma 7. ([7]) Let $\mathfrak{p}$ be a 4-dimensional real vector spaces, $\mathfrak{h}$ a real vector space of dimension three, and let $[\cdot, \cdot]: \mathfrak{p} \times \mathfrak{p} \rightarrow \mathfrak{h}$ be a skew-symmetric bilinear map such that $i_{X} \circ[\cdot, \cdot]: \mathfrak{p} \rightarrow \mathfrak{h}$ is onto for all $X \neq 0 \in \mathfrak{p}$. Then, fixing a basis of $\mathfrak{p}$ the function $\Omega: \mathfrak{h}^{\star} \rightarrow I R$ defined by $\Omega(f)=\sqrt{\operatorname{det}(f \circ[\cdot, \cdot])}$ is a positive definite quadratic form in $\mathfrak{h}^{\star}$, determined by $[\cdot, \cdot]$ up to a positive scale factor.

Proof. From Lemma 6 we know that $(f \circ[\cdot, \cdot])$ is a non-degenerate skew-symmetric bilinear form, for any nonzero functional $f \in W^{*}$. Thus, the matrix of $(f \circ[\cdot, \cdot])$ in a fixed basis $\beta=\left\{e_{1}, e_{2}, e_{3}, e_{4}\right\}$ of $\mathfrak{p}$ is a skew-symmetric matrix given by

$$
[f \circ[\cdot, \cdot]]_{\beta}=\left(\begin{array}{cccc}
0 & a_{12} & a_{13} & a_{14} \\
-a_{12} & 0 & a_{23} & a_{24} \\
-a_{13} & -a_{23} & 0 & a_{34} \\
-a_{14} & -a_{24} & -a_{34} & 0
\end{array}\right)
$$

where $a_{i j}=f \circ\left[e_{i}, e_{j}\right] ; i=1,2,3 ; j=2,3,4$ and $i<j$. Then,

$$
\operatorname{det}[f \circ[\cdot, \cdot]]_{\beta}=\left(a_{12} a_{34}-a_{13} a_{24}+a_{23} a_{14}\right)^{2} .
$$

Consequently, $\forall a \in I R$ we have that

$$
\Omega(a f)=\sqrt{\operatorname{det}[a f \circ[\cdot, \cdot]]_{\beta}}=\sqrt{a^{4} \operatorname{det}[f \circ[\cdot, \cdot]]_{\beta}}=a^{2} \sqrt{\operatorname{det}[f \circ[\cdot, \cdot]]_{\beta}}=a^{2} \Omega(f),
$$

in other words, $\Omega$ is a quadratic form. Also, since that for any nonzero functional $f \in W^{*}$, the matrix $[f \circ[\cdot, \cdot]]_{\beta}$ is invertible and by hypothesis $i_{X} \circ[\cdot, \cdot]$ is onto, it follows that $\Omega$ is positive definite. 
To see that $\Omega$ is determined by $[\cdot, \cdot]$ up to a positive scale factor it is enough to observe that if $\beta^{\prime}=\left\{e_{1}{ }^{\prime}, e_{2}{ }^{\prime}, e_{3}{ }^{\prime}, e_{4}{ }^{\prime}\right\}$ is another basis of $\mathfrak{p}$ then,

$[f \circ[\cdot, \cdot]]_{\beta^{\prime}}=G^{t}[f \circ[\cdot, \cdot]]_{\beta} G$, where $G$ is the change of basis matrix from $\beta^{\prime}$ to $\beta$, and as a consequence $\operatorname{det}[f \circ[\cdot, \cdot]]_{\beta^{\prime}}=(\operatorname{det} G)^{2} \operatorname{det}[f \circ[\cdot, \cdot]]_{\beta}$.

Theorem 6. ([7]) Let $\mathfrak{p}$ be a 4-dimensional real vector space, $\mathfrak{h}$ a real vector space of dimension three, and let $[\cdot, \cdot]: \mathfrak{p} \times \mathfrak{p} \rightarrow \mathfrak{h}$ be a skew-symmetric bilinear map such that $i_{X} \circ[\cdot, \cdot]: \mathfrak{p} \rightarrow \mathfrak{h}$ is onto for all $X \neq 0 \in \mathfrak{p}$. Then, the 2-step nilpotent Lie algebra $\mathfrak{g}=\mathfrak{p} \oplus \mathfrak{h}$ where the bracket of vectors on $\mathfrak{g}$ is the extension of the bilinear map $[\cdot, \cdot]$ given by $[\mathfrak{p}, \mathfrak{h}]=0,[\mathfrak{h}, \mathfrak{h}]=0$ is isomorphic to the 7 -dimensional quaternionic Heisenberg algebra.

Proof. Let $\{A, B, C\}$ be the dual basis of a basis of $\mathfrak{h}^{*}$ that orthonormalizes the positive definite quadratic form $\Omega$ defined in Lemma 7 , up to a positive scale factor. Take any nonzero vector $U_{1} \in \mathfrak{p}$ and then, from the sobrejectivity of $i_{U_{1}} \circ[\cdot, \cdot]: \mathfrak{p} \rightarrow \mathfrak{h}$ there exist $U_{2}, U_{3}$ and $U_{4} \in \mathfrak{p}$ such that $\left[U_{1}, U_{2}\right]=A,\left[U_{1}, U_{3}\right]=B$ and $\left[U_{1}, U_{4}\right]=C$. Observe that $\left\{U_{1}, U_{2}, U_{3}, U_{4}\right\}$ is a basis of $\mathfrak{p}$. Now, write $[\cdot, \cdot]=\mathcal{L}_{1} A+\mathcal{L}_{2} B+\mathcal{L}_{3} C$ where $\mathcal{L}_{i}: \mathfrak{p} \times \mathfrak{p} \rightarrow I R, i=1,2,3$; and then, set

$$
\begin{aligned}
& V_{2}=U_{2}-\mathcal{L}_{2}\left(U_{2}, U_{3}\right) U_{1}, \\
& V_{3}=U_{3}+\mathcal{L}_{1}\left(U_{2}, U_{3}\right) U_{1} \quad \text { and } \\
& V_{4}=U_{4}+\mathcal{L}_{1}\left(U_{2}, U_{4}\right) U_{1} .
\end{aligned}
$$

We obtain the following relations:

$$
\begin{aligned}
{\left[U_{1}, V_{2}\right] } & =A, \quad\left[U_{1}, V_{3}\right]=B, \quad\left[U_{1}, V_{4}\right]=C, \\
{\left[V_{2}, V_{3}\right] } & =\mathcal{L}_{3}\left(U_{2}, U_{3}\right) C=\delta C, \\
{\left[V_{2}, V_{4}\right] } & =\mathcal{L}_{2}\left(U_{2}, U_{4}\right) B+\left[\mathcal{L}_{3}\left(U_{2}, U_{4}\right)-\mathcal{L}_{2}\left(U_{2}, U_{3}\right)\right] C=\beta B+\varepsilon C, \\
{\left[V_{3}, V_{4}\right] } & =\mathcal{L}_{1}\left(U_{3}, U_{4}\right) A+\left[\mathcal{L}_{2}\left(U_{3}, U_{4}\right)-\mathcal{L}_{1}\left(U_{2}, U_{4}\right)\right] B \\
& +\left[\mathcal{L}_{3}\left(U_{3}, U_{4}\right)+\mathcal{L}_{1}\left(U_{2}, U_{3}\right)\right] C=\alpha A+\gamma B+\zeta C .
\end{aligned}
$$

Then, in the basis $\left\{U_{1}, V_{2}, V_{3}, V_{4}\right\}$ of $\mathfrak{p}$ we have

$$
\begin{array}{r}
\operatorname{det}\left[x\left(\begin{array}{cccc}
0 & 1 & 0 & 0 \\
-1 & 0 & 0 & 0 \\
0 & 0 & 0 & \alpha \\
0 & 0 & -\alpha & 0
\end{array}\right)+y\left(\begin{array}{cccc}
0 & 0 & 1 & 0 \\
0 & 0 & 0 & \beta \\
-1 & 0 & 0 & \gamma \\
0 & -\beta-\gamma & 0
\end{array}\right)+z\left(\begin{array}{cccc}
0 & 0 & 0 & 1 \\
0 & 0 & \delta & \varepsilon \\
0 & -\delta & 0 & \zeta \\
-1 & -\varepsilon-\zeta & 0
\end{array}\right)\right]= \\
\left(\alpha x^{2}-\beta y^{2}+\delta z^{2}+\gamma x y+\zeta x z-\varepsilon y z\right)^{2},
\end{array}
$$


for arbitrary real $x, y, z$.

On the other hand,

$$
\begin{aligned}
\left(x \mathcal{L}_{1}+y \mathcal{L}_{2}+z \mathcal{L}_{3}\right) & =\left(x A^{*}+y B^{*}+z C^{*}\right) \circ\left(\mathcal{L}_{1} A+\mathcal{L}_{2} B+\mathcal{L}_{3} C\right) \\
& =\left(x A^{*}+y B^{*}+z C^{*}\right) \circ[\cdot, \cdot]
\end{aligned}
$$

where $\left\{A^{*}, B^{*}, C^{*}\right\}$ is the dual basis of the basis $\{A, B, C\}$. Then, we also have

$$
\begin{aligned}
\operatorname{det}\left(x \mathcal{L}_{1}+y \mathcal{L}_{2}+z \mathcal{L}_{3}\right) & =\operatorname{det}\left[\left(x A^{*}+y B^{*}+z C^{*}\right) \circ[\cdot, \cdot]\right] \\
& =\left[\Omega\left(x A^{*}+y B^{*}+z C^{*}\right)\right]^{2}=\lambda\left(x^{2}+y^{2}+z^{2}\right)^{2}
\end{aligned}
$$

for some $\lambda>0$, since $\left\{A^{*}, B^{*}, C^{*}\right\}$ is the basis of $\mathfrak{h}^{*}$ that orthonormalizes the positive definite quadratic form $\Omega$, up to a positive scale factor.

So, the equality

$$
\left(\alpha x^{2}-\beta y^{2}+\delta z^{2}+\gamma x y+\zeta x z-\varepsilon y z\right)^{2}=\lambda\left(x^{2}+y^{2}+z^{2}\right)^{2},
$$

is verified for any real $x, y, z$ and for some $\lambda>0$. This implies that $\alpha=-\beta=\delta \neq 0$ and $\gamma=\zeta=\varepsilon=0$.

Thus, the transformation defined by

$$
\begin{aligned}
X_{1} & =U_{1}, & & Z_{1}=\frac{1}{\alpha} A, \\
Y_{1} & =\frac{1}{\alpha} V_{2}, & Z_{2} & =\frac{1}{\alpha} B, \\
V_{1} & =\frac{1}{\alpha} V_{3}, & Z_{3} & =\frac{1}{\alpha} C \\
W_{1} & =\frac{1}{\alpha} V_{4}, & &
\end{aligned}
$$

is an isomorphism onto the 7-dimensional quaternionic Heisenberg algebra.

Corollary 4. The only nilpotent sub-OIL fat algebra $(\mathfrak{g}=\mathfrak{p} \oplus \mathfrak{h}, s, \mathfrak{k}=0, B)$ with $\mathfrak{h}=[\mathfrak{p}, \mathfrak{p}]$, dim $\mathfrak{g}=7$ and dim $\mathfrak{h}=3$ is the quaternionic Heisenberg algebra (see Example 2).

Remark 5. The Corollary 4 is not true if we replace 7 -dimensional to 11 -dimensional; see some counterexamples in [16].

Acknowledgements. This paper was written while the first author was a CAPES Posdoctoral fellow (Grant 6124/12-7) at Institut de Mathématiques d'Université Pierre et Marie Curie, Paris VI. She thanks L'équipe d'Analyse Complexe et Géométrie for their kind hospitality. The second author was supported in part by the ANR through the project Structure Géométriques et Triangulations. 


\section{References}

1. Almeida, D. M., On fat sub-Riemannian symmetric spaces in codimension three, Differential Geom. Appl. 24 (2006), 178-190.

2. Bellaiche, A., The tangent space in sub-Riemannian geometry, in Sub-Riemannian Geometry, Series Progress in Mathematics 144, pp. 1-78, Birkhäuser, Basel, 1996.

3. Berndt, J., Tricerri, F. and Vanhecke, L., Generalized Heisenberg Groups and Damek-Ricci Harmonic Spaces, Lecture Notes in Math., p. 1598, Springer, Berlin, 1995.

4. Berndt, J., Ricci, F. and Vanhecke, L., Weakly symmetric groups of Heisenberg type, Differential Geom. Appl. 8 (1998), 275-284.

5. Bieliavsky, P., Falbel, E. and Gorodski, C., The classification of simply-connected contact sub-Riemannian symmetric spaces, Pacific J. Math. 188 (1999), 65-81.

6. Bröcker, T. and том Dieck, T., Representations of Compact Lie Groups, Graduate Texts in Matematics 98, Springer, Berlin, 1985.

7. Derdzinski, A. and Rigas, A., Unflat connections in 3-sphere bundles over $S^{4}$, Trans. Amer. Math. Soc. 265 (1981), 485-493.

8. Eberlein, P., Geometry of two-step nilpotent Lie groups with a left invariant metric, Ann. Sci. Éc. Norm. Supér. 27 (1994), 611-660.

9. Eberlein, P. and HeBer, J., Quarter pinched homogeneous spaces of negative curvature, Internat. J. Math. 7 (1996), 441-500.

10. Falbel, E. and Gorodski, C., On contact sub-Riemannian symmetric spaces, Ann. Sci. Éc. Norm. Supér. 28 (1995), 571-589.

11. Gauger, M., On the classification of metabelian Lie algebras, Trans. Amer. Math. Soc. 179 (1973), 293-329.

12. Gordon, C. S., Naturally reductive homogeneous Riemannian manifolds, Canad. J. Math. 37 (1985), 467-487.

13. Kaplan, A., On the geometry of groups of Heisenberg type, Bull. Lond. Math. Soc. 15 (1983), 35-42.

14. Kobayashi, S. and Nomizu, K., Foundations of Differential Geometry, Vol. II, WileyInterscience, New York, 1969.

15. Lauret, J., Homogeneous nilmanifolds attached to representations of compact Lie groups, Manuscripta Math. 99 (1999), 287-309.

16. Lauret, J. and Oscari, D., On Nonsingular two-step nilpotent Lie algebras (2012). arXiv: 1209. 3060v1 [math.RA].

17. Montgomery, R., A survey of syngular curves in sub-Riemannian geometry, J. Dyn. Control Syst. 1 (1995), 49-90.

18. Strichartz, R., Sub-Riemannian geometry, J. Differential Geom. 24 (1986), 221263.

19. Wolf, J. A., Spaces of Constant Curvature, 6th ed., Chelsea, New York, 2011.

D.M. Almeida

Faculdade de Matemática da Universidade

Federal de Uberlandia

Uberlandia

Brazil

dulce.almeida@ufu.br 
E. Falbel

Institut de Mathématiques de Jussieu

Université Pierre et Marie Curie

Paris

France

and

Ouragan-INRIA

Paris

France

elisha.falbel@prg-imj.fr

Received February 27, 2016

in revised form October 24, 2017 